\title{
Static shift levelling using geomagnetic transfer functions
}

\author{
Juanjo Ledo ${ }^{1}$, Anna Gabàs ${ }^{2}$, and Alex Marcuello² \\ ${ }^{1}$ Geological Survey of Canada, 615 Booth Street, Ottawa, Ontario, K1A 0E9 Canada \\ ${ }^{2}$ Departament de Geodinàmica i Geofisica, Universitat de Barcelona, Spain \\ (Received December 14, 2000; Revised March 27, 2001; Accepted March 28, 2001)
}

\begin{abstract}
Galvanic distortion of magnetotelluric (MT) data is a common problem in the study of the Earth's electrical properties. These distortions are local, they affect independently each MT site, and where restricted to distortion of the electric field are manifest as vertical shifts in the apparent resistivity curves (static shift). The removal of the static shift is necessary to avoid misinterpreting MT data. We present a method that allows us to partially retrieve the regional response of the TE-mode data in a 2D case. The method determines relative changes between distortion parameters along a profile, and is based in the Faraday's law, and uses only magnetotelluric responses: measured impedance tensor and geomagnetic transfer function (tipper). The method is valid under the assumption that the variation of horizontal magnetic field can be neglected, and a test for checking this criterion has been developed. The mathematics involved in the procedure are straightforward, and can be stated as a linear regression. We present successful applications to both synthetic and real (COPROD data) datasets.
\end{abstract}

\section{Introduction}

The build up of charge near inhomogeneities causes electric field redistribution, and results in galvanic distortions affecting the regional response in magnetotelluric (MT) data. It has been shown that the regional electric field and the local electric field caused by the accumulation of charges in the inhomogeneity are in phase and frequency independent (Bahr, 1988; Jiracek, 1990; Chave and Smith, 1994). Thus, if the regional structure is $1 \mathrm{D}$, the galvanic distortion will manifest itself as a frequency-independent displacement of the measured apparent resistivity (static shift) in comparison with the undistorted case. In the $2 \mathrm{D}$ case, if the data have been rotated to the principal axes, the effect will be the same as in $1 \mathrm{D}$. However, if the regional structure is $3 \mathrm{D}$, both, the measured apparent resistivity and the phases, are distorted in a frequency-dependent manner (Ledo et al., 1998). Different methodologies have been proposed to remove or, at least, to reduce the static shift effect; Ogawa (2000) and references therein presented a recent review. One of the newest is the use of Faraday's law as a physical constraint. The use of Faraday's law allows us to relate the horizontal derivative of the distorted impedance tensor with the undistorted geomagnetic transfer function. Faraday's law was used by Chouteau et al. (1996), to propose a transformation filter for VLF that converts the tipper vector into an apparent resistivity estimate by assuming that the spatial derivatives of the magnetic field horizontally can be neglected. Gharibi and Pedersen (1999) improved this method by proposing an iterative method to estimate the impedance from magnetic field measurements by making use of the fact that the secondary horizontal and vertical magnetic fields are of internal origin. Such methods were designed to obtain the impedance tensor

Copy right (c) The Society of Geomagnetism and Earth, Planetary and Space Science (SGEPSS); The Seismological Society of Japan; The Volcanological Society of Japan; The Geodetic Society of Japan; The Japanese Society for Planetary Sciences. from the magnetic fields, and not to correct the galvanic distortion. Utada and Munekane (1999) proposed a method to correct the galvanic distortion of the MT impedance in cases where the regional structure is $3 \mathrm{D}$ by using the impedance tensor, the horizontal magnetic components, and their horizontal gradients. These authors pointed out the possible use of Faraday's law to recover the regional TE apparent resistivity over a 2D regional model. Garcia and Jones (1999) proposed a method to correct galvanic distortion on 3D environments, assuming that two neighboring sites sense the same regional structure. However, the methods proposed by Utada and Munekane (2000) and Garcia and Jones (1999) are, in general, only applicable to surveys with simultaneous recording at several sites and with small station spacing respectively.

We present a methodology to recover the regional TEmode response over a $2 \mathrm{D}$ regional earth using only the measured electromagnetic (EM) response functions: impedance and geomagnetic transfer functions, not the EM fields. We will follow the approach suggested by Chouteau et al. (1996), whereby the spatial derivatives of the magnetic field are neglected. Thus, the main goals of this work are two: 1) determine when the horizontal derivative of the magnetic field is negligible, and then, 2) correct the TE-mode apparent resistivity from galvanic distortions. We develop firstly the theory to justify our approach, and then, we show its applicability presenting two examples, one with synthetic data, and another with real data.

\section{Theory}

The expression relating the measured MT components (impedance and geomagnetic transfer functions) can be easily derived from Faraday's law as follows:

$$
\operatorname{rot} \vec{E}=-i \omega \mu \vec{H}
$$




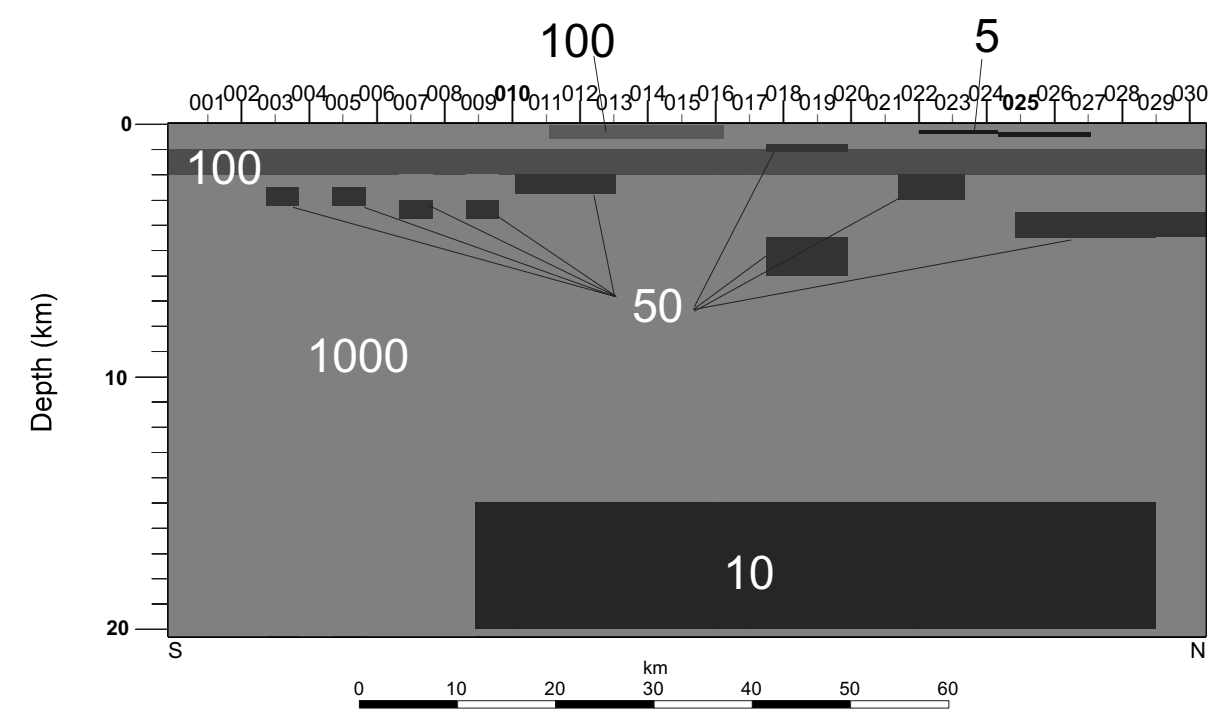

Fig. 1. The 2D model used for generating the synthetic data. It contains different conductive anomalies at several scales. Resistivities are in $\Omega$.m.

Where a time dependence of the type $\exp (+i \omega t)$ has been assumed for the EM fields. Considering $x$ the strike direction, for the TE-mode of a 2D structure, the vertical component of Eq. (1) is:

$$
\frac{\partial E_{x}}{\partial y}=i \omega \mu H_{z}
$$

Now the TE-mode electric field can be expressed as the product of the impedance element and the magnetic field $\left(E_{x}=Z_{x y} H_{y}\right)$, and the vertical magnetic field can be expressed as $H_{z}=T_{y} H_{y}$ that brings us to the expression:

$$
\frac{\partial Z_{x y}}{\partial y}+\frac{Z_{x y}}{H_{y}} \frac{\partial H_{y}}{\partial y}=i \omega \mu T_{y},
$$

and integrating along the $y$ domain, we obtain:

$$
\Delta Z_{x y}=i \omega \mu<T_{y}>\Delta y-<Z_{x y}>\Delta \ln H_{y}
$$

where $<\cdot>$ denotes the mean value over the integration domain.

At this point, depending on the way the data were acquired, there are at least three possible paths to follow. 1) When the MT responses have been obtained using an array where all the components have been measured simultaneously at all sites then, the variation of the $H_{y}$ field can be calculated; this method requires a large number of acquisition systems available to record the data simultaneously. Usually, only a few acquisition systems are available, and the simultaneous field variations cannot be determined. 2) When simultaneous recording was not possible, Gharibi and Pedersen (1999) calculated the horizontal magnetic field distribution through an iterative process to obtain the full solution. These authors made use of the fact that the horizontal and vertical components of the secondary magnetic can be related through a Hilbert transform pair in the wavenumber domain. However, to ensure a good estimate of the horizontal magnetic field, a long profile and a dense array of stations are required. 3) The approach we have adopted in this work

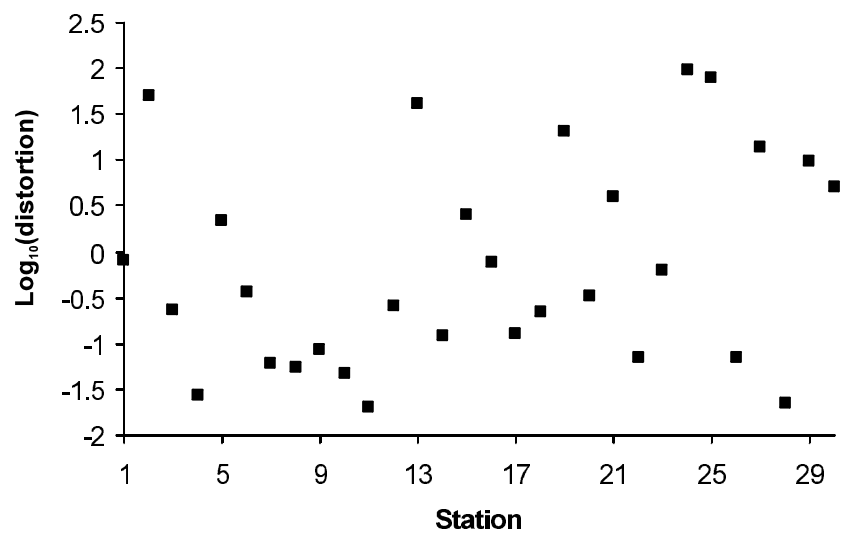

Fig. 2. Distortion factor applied to each synthetic site.

follows the one proposed by Chouteau et al. (1996). These authors proposed to obtain the apparent resistivity distribution for the VLF-EM data neglecting the changes of the horizontal magnetic field.

Under this approximation Eq. (3) can be written as:

$$
\Delta Z \cong i \omega \mu<T>\Delta y
$$

where the subscripts $x y$ and $y$ have been omitted in order to simplify the notation (where there is no confusion, we will use this convention). Using the trapezoid rule for the integration, and taking two consecutive sites $j$ and $j-1$, we can express the impedance at site $j$ as a function of the impedance at site $j-1$ and the geomagnetic transfer functions at both sites as:

$$
\Delta Z_{j} \cong Z_{j-1}+i \omega \mu \frac{\Delta y}{2}\left(T_{j}+T_{j-1}\right)
$$

We can now formulate a relationship between transfer functions $Z$ and $T$. In the previous development, we have considered that these functions are regional functions (unaffected by galvanic distortion). Nevertheless, if galvanic 

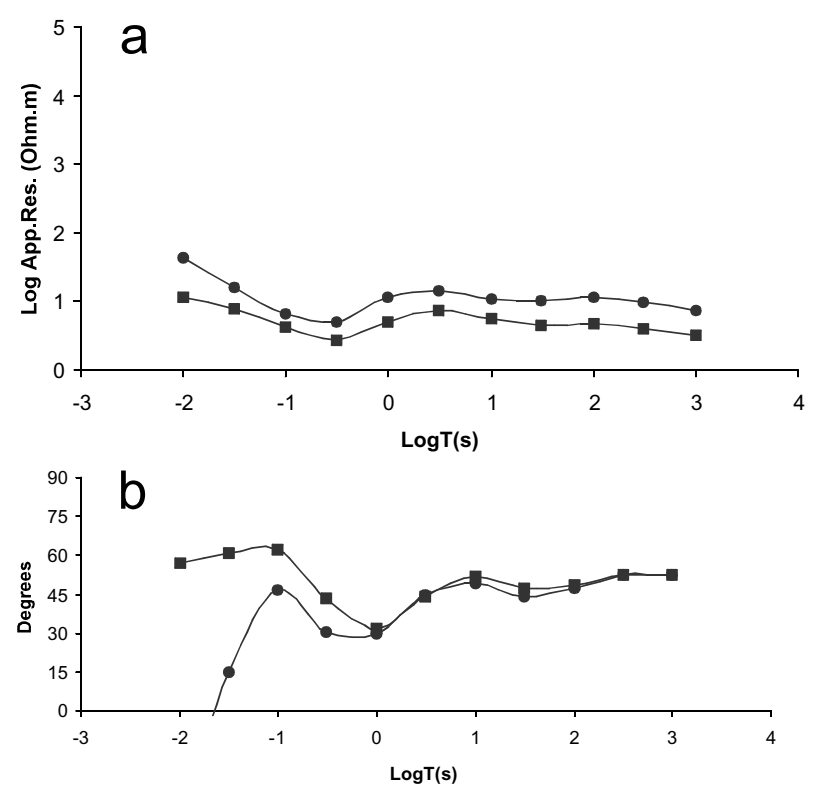

Fig. 3. Distorted data and calculated data using Eq. (5) for site 10; a) TE-mode apparent resistivities, b) TE-mode phases. Black squares: distorted data from the model. Black Circles: values obtained from Eq. (5), see text.

distortions are present, the quotient between the distorted impedance, $Z^{M}$, and the regional impedance, $Z$, will be a real and frequency independent number $a$.

$$
\frac{Z_{j}^{M}}{a_{j}} \cong \frac{Z_{j-1}^{M}}{a_{j-1}}+i \omega \mu \frac{\Delta y}{2}\left(T_{j}+T_{j-1}\right)
$$

Dividing by the second term on the right side, and rearranging using the following change of variables $\zeta(\omega)=$ $Z_{j}^{M} /\left(i \omega \mu \Delta y\left(T_{j}+T_{j-1}\right) / 2\right)$, and $\psi(\omega)=Z_{j-1}^{M} /(i \omega \mu \Delta y$ $\left.\left(T_{j}+T_{j-1}\right) / 2\right)$ :

$$
\zeta(\omega)=\frac{a_{j}}{a_{j-1}} \psi(\omega)+a_{j}
$$

Formula (7) describes a straight line in the $\zeta \psi$-space, where the points $(\zeta, \psi)$ depend on the frequency. So, the determination of the distortion parameters can be understood as a line fitting problem. If the requirement for the slow variation of the horizontal magnetic fields fails, Eq. (7) will not be valid. The description given by (7) is only valid in a range of frequencies; however, the method we propose allows us to delimit this range of frequencies, and obtain information about the distortion parameters $a_{j}$ and $a_{j-1}$ from the line fitting. In this range of frequencies, this condition implies that the regional and measured TE apparent resistivity curves must be parallel and their phases curves must coincide. As we will show in the examples section this criterion allows to gain insight into the method.

Our procedure is stated as follows, we chose sets of points $(\zeta, \psi)$ covering different ranges of frequencies, and we test if these sets describe a straight line in the $\zeta \psi$-space. To do so, we check the correlation index, the slope and independent term in (6), and the residual $\epsilon$, which gives a "measure' of the quality in the approximation. The following formula can be inferred from expression (3), after applying the
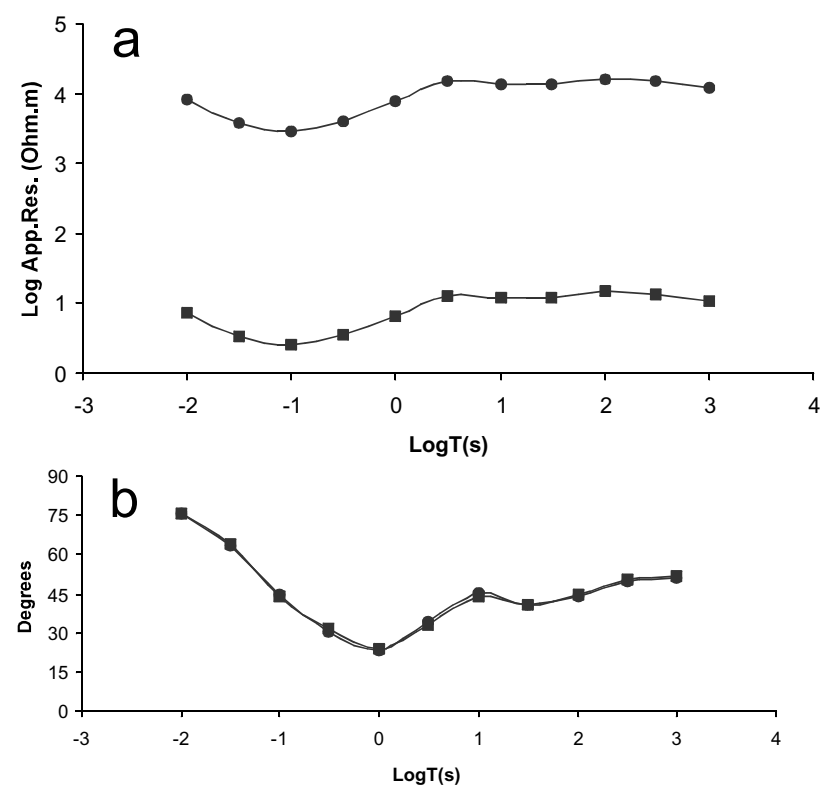

Fig. 4. Distorted data and calculated data using Eq. (5) for site 25; a) TE-mode apparent resistivities, b) TE-mode phases. Black squares: distorted data from the model. Black circles: values obtained from Eq. (5), see text.

changes used to obtain (6);

$$
\begin{aligned}
\epsilon & =\frac{<Z>\Delta \ln H}{i \omega \mu \Delta y\left(T_{j}+T_{j-1}\right) / 2} \\
& \cong \frac{\Delta \ln H}{\Delta \ln H+\Delta Z /<Z>} \approx\left(1+\frac{\Delta \ln Z}{\Delta \ln H}\right)^{-1}
\end{aligned}
$$

The residual $\epsilon$ checks if the changes of $H$ can be neglected relative to the changes of $Z$. The criteria followed to chose the distortion parameters have been: the highest correlation index, the lowest residual, and additionally the existence of positive parameters in (7), because distortion parameters must be positive. In this procedure, the slope of the regression is well determined, but not the independent term. This implies that only relative rates between distortion parameters (static shifts) will be fully determined, and the determination of the distortion parameters will be necessary in a single station. Although the absolute leveling of the apparent resistivity it is not fully obtained with this technique, the result of Eq. (5) is important, because it allows us to determine the relative leveling between sites.

\section{Synthetic Model}

In this section a numerical example shows the suitability of the proposed method. We have calculated the MT response (impedance and transfer function) of a 2D model (Fig. 1) using the code of Wannamaker et al. (1986). This model includes several conductive anomalies at different scales that will allow us to test our hypothesis about the variation of the horizontal magnetic field. The period band used is between $100 \mathrm{~Hz}$ and $1000 \mathrm{~s}$, and the sites are located every $3 \mathrm{~km}$. The mesh used was of 400 horizontal elements and 120 vertical elements. Subsequently, each one of the apparent resistivity curves for the TE-mode was distorted 


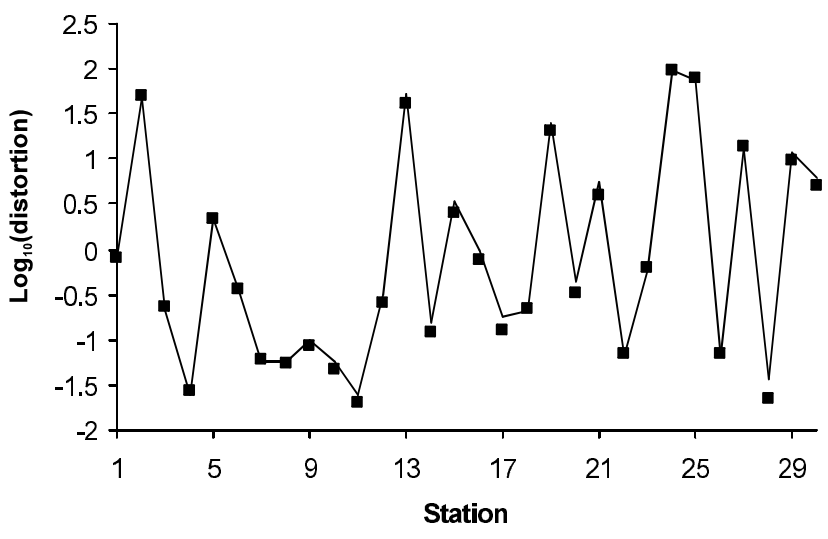

Fig. 5. Comparison of the applied static shift to that calculated with our technique. Continuous line: values obtained with the method proposed in this work. Black squares: values applied to the response from model of Fig. 1.

by a random factor between -2 and 2 in logarithmic scale (Fig. 2), and they were taken as "measured" data.

To investigate the validity of our approximation, we compare the measured apparent resitivity and phases with the computed values from Eq. (5). Figures 3 and 4 show the "measured" apparent resistivity and phase curves compared with the values computed with Eq. (5) for sites 10 and 25; black squares represents the distorted data used, and black circles the values calculated from (5). We can see in Fig. 3 that the calculated phases are not coincident with the measured ones for short periods, and the apparent resistivity
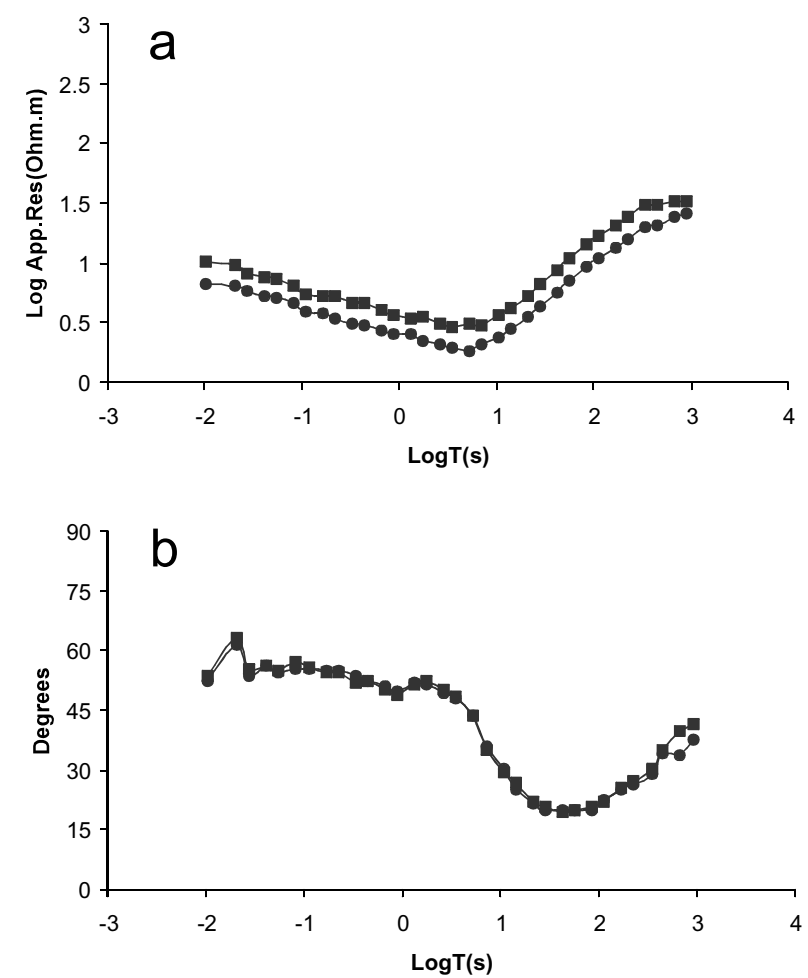

Fig. 6. Raw and calculated data using Eq. (5) for site 005; a) TE-mode apparent resistivities, b) TE-mode phases. Black squares: raw data. Black circles: values obtained from Eq. (5), see text. curves are not parallel. This observation indicates that the variation of the horizontal magnetic fields cannot be neglected for short periods at this site. To calculate the relative static shift between the distorted data and that obtained through Eq. (7), only the long period data are valid and were used for this site. On the other hand, Fig. 4 shows an example where the calculated data agree with distorted data; in this case all the available data were used to calculate the distortion factor.

We have applied Eq. (7) to the distorted data, choosing station 1 as our reference site. The election of the reference site is arbitrary in this case. When working with real data the reference site must be chosen with care, because as we said earlier the values obtained by applying Eq. (7) will be affected by the distortion of the reference site. We can see the agreement between the values of the applied distortion (solid line) with the values of the calculated distortion referenced to station 1 (black squares) in Fig. 5. As can be observed the result of the method determines adequately the distortion parameters.

\section{Real Data}

In the previous section we have seen that the method, when neglecting the horizontal variations of the magnetic field, can be applied even when the $2 \mathrm{D}$ model presents moderate to strong conductivity contrasts at different scale lengths. For the second example, we have chosen part of the well-known COPROD2R dataset (Jones, 1993) acquired in southern Saskatchewan and Manitoba (Canada). A major conductive anomaly, the North American Central Plains
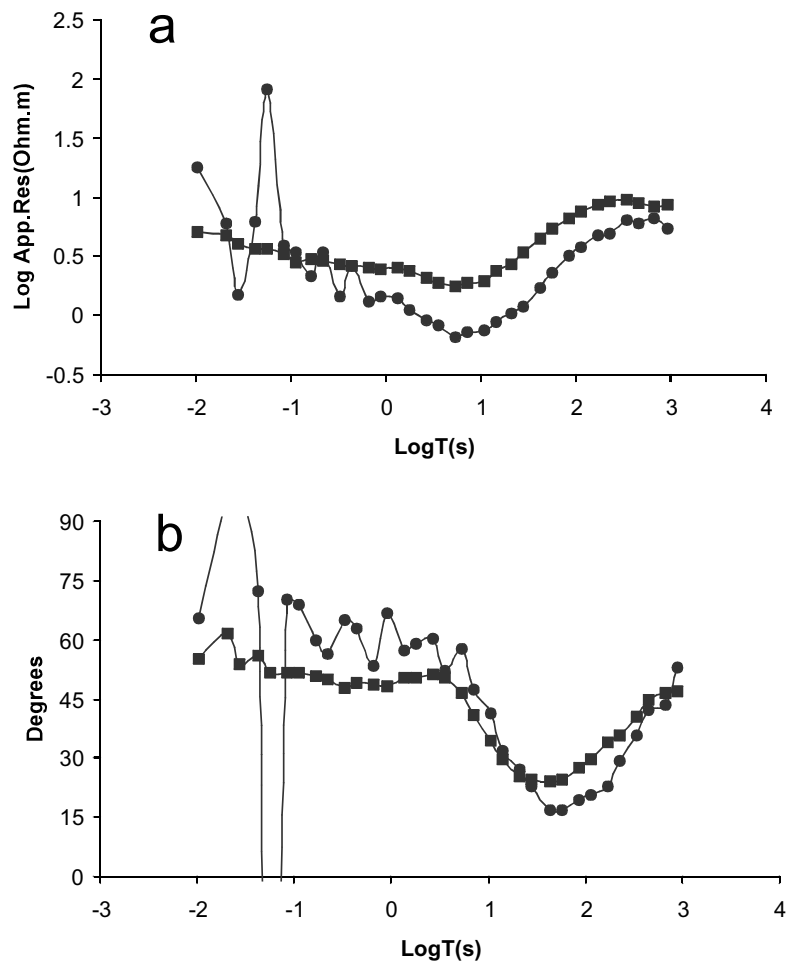

Fig. 7. Raw data and calculated data using Eq. (5) for site 002; a) TE-mode apparent resistivities, b) TE-mode phases. Black squares: raw data. Black circles: values obtained from Eq. (5), see text. 


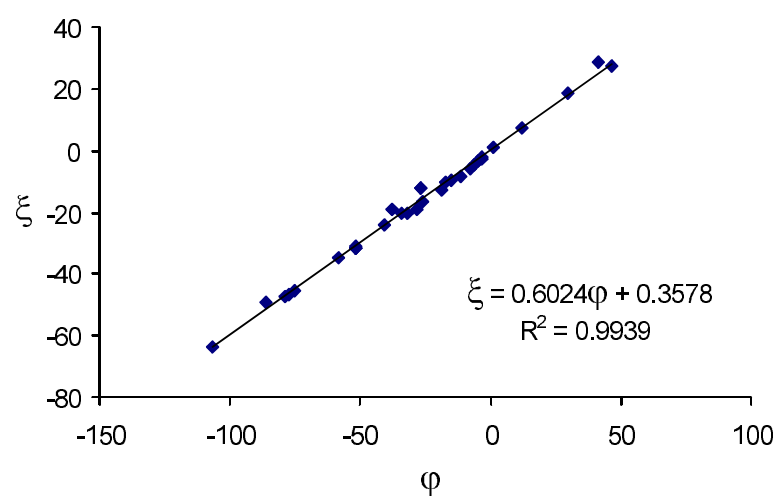

Fig. 8. Values of $\zeta(\omega)$ and $\psi(\omega)$ for the entire period range for site 005 , and linear regression results.
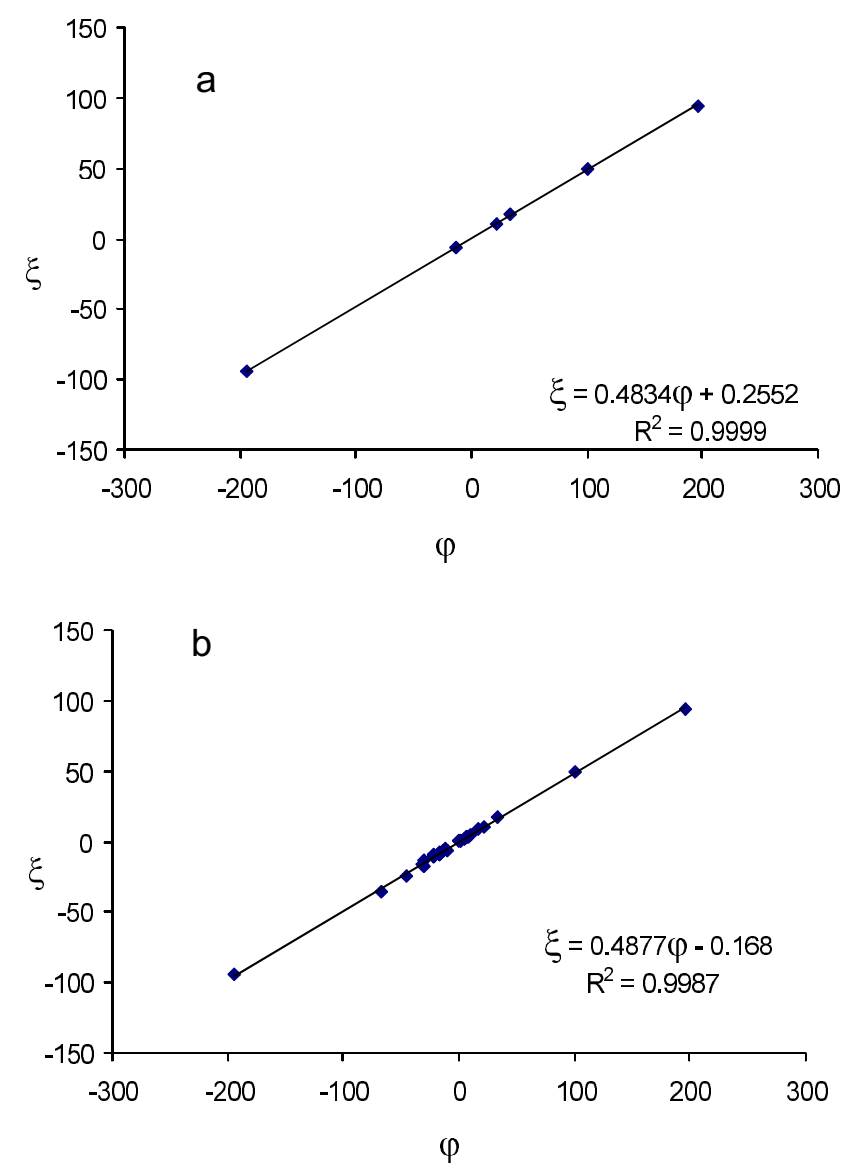

Fig. 9. (a) Values $\zeta(\omega)$ and $\psi(\omega)$ for the period range $8-30 \mathrm{~s}$ for site 002 and linear regression results. (b) Values $\zeta(\omega)$ and $\psi(\omega)$ for the whole period range for site 002 and linear regression results.

(NACP) anomaly, was detected using this data (Jones and Savage, 1986) with a NS strike direction. This dataset is an excellent framework to apply the method described in the previous section because of the high quality of the data, the well defined two-dimensionality of the data, and the information from well-logs used by Jones (1988) to correct the static shift distortion.

As we did for the synthetic data we compare the measured data with those calculated from the tipper following

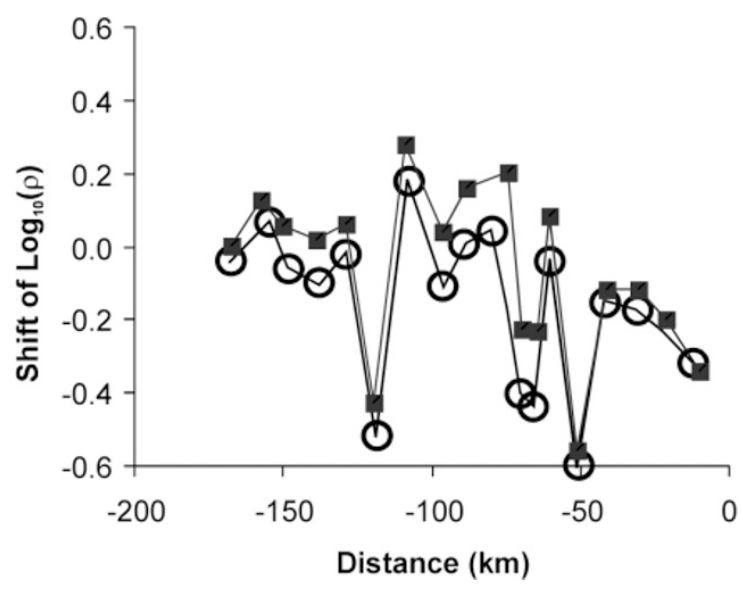

Fig. 10. Comparison of the static shift coefficients determined by Jones (1988), circles, to the values obtained from the application of our method, black squares.

the Eq. (5). Apparent resistivities and phases are shown in Figs. 6 and 7 for sites 005 and 002 respectively. We can see in Fig. 6 the agreement for phases, and apparent resistivity curves are parallel in all the period range. In this case, the requirement for slow spatial variation of the magnetic fields horizontally has been met for the whole period range. Figure 8 shows the values $\zeta(\omega)$ and $\psi(\omega)$ for all periods and the line fitting. On the other hand, Fig. 7 indicates that for site 002 the approximation of slow variation of the horizontal magnetic field is only valid for a narrow period band of 8-30 s. To calculate the relative static shift between the distorted data and the obtained through Eq. (6) only the data in this period-band was used for site 002. Figure 9(a) shows the values $\zeta(\omega)$ and $\psi(\omega)$ for this period range and the results of the linear regression. Figure 9(b) shows the values $\zeta(\omega)$ and $\psi(\omega)$ for the whole period range, it can be observed that the independent term is negative which violates the definition of positive distortion parameters.

After the selection of the frequency band for each site, we can obtain the relative static shift between continuous stations. Thereafter we choose one of the sites as reference, and the relative static shift has been recalculated from this site. In our case we choose the westernmost station as reference site. Figure 10 shows the comparison between the static shift coefficients calculated by Jones (1988), and by the method presented here. The maximum difference between the two methods is a factor of only 1.5 .

\section{Conclusions}

The use of Faraday's law permits the retrieval of the relative leveling of the static shift affecting the TE-mode component of a 2D structure using only MT data. The method presented here is based on the approximation that small spatial variations of the magnetic fields horizontally can be neglected. The synthetic data show that the approximation is valid for moderate to strong conductivity contrasts at different scale lengths. Moreover, the method allows testing the validity of the approximation in a frequency range. We have applied this method to real data (COPROD dataset), and the comparison with the corrected data by Jones (1988) that us- 
ing well-log information shows good agreement. This technique retrieves the relative static shift between sites, but this is a strong constraint on the data. The absolute static shift needs to be determined at a single station; in such situation, it can be applied to the inversion schemes but with only one unknown value, that will be need to fix the static shift for all the sites. This technique offers a reliable alternative to the other known techniques.

Acknowledgments. We would like to acknowledge P. J. Savage of PanCanadian Petroleum Limited for making the raw (uncorrected) data available to the GSC, and to A. G. Jones for making the corrected data available. A. G. Jones, P. Queralt, J. Craven, X. Garcia and R. Kurtz provided helpful comments on this work. The manuscript has benefited from reviews by V. Cerv and F. E. M. Lilley, we also want to thank the Guest Editor A. L. Padilha. Geological Survey of Canada Contribution number 200293.

\section{References}

Bahr, K., Interpretation of the magnetotelluric impedance tensor: regional induction and local telluric distortion, J. Geophys., 62, 119-127, 1988.

Chave, A. D. and J. T. Smith, On electric and magnetic galvanic distortion tensor decomposition, J. Geophys. Res., 99, B3, 4669-4682, 1994.

Chouteau, M., P. Zhang, and D. Chápellier, Computation of apparent resistivity profiles from VLF-EM data using linear filtering, Geophysical Prospecting, 44, 215-232, 1996.
Garcia, X. and A. G. Jones, Extended decomposition of MT data, in The second international symposium on Three-dimensional Electromagnetics (3DEM-2), Salt Lake City (USA), 1999.

Gharibi, M. and L. B. Pedersen, Transformation of VLF data into apparent resistivities and phases, Geophysics, 64, 1393-1402, 1999.

Jiraceck, G., Near surface and topographic distortion in electromagnetic induction, Surveys in Geophysics, 11, 163-203, 1990.

Jones, A. G., Static shift of magnetotelluric data and its removal in a sedimentary basin environment, Geophysics, 53, 967-978, 1988.

Jones, A. G., The COPROD2 dataset: Tectonic setting, recorded MT data, and comparison of models, J. Geomag. Geoelectr., 45, 933-955, 1993.

Jones, A. G. and J. G. Savage, North American Central Plains conductivity anomaly goes east, Geophys. Res. Lett., 13, 685-688, 1986.

Ledo, J., P. Queralt, and J. Pous, Galvanic Distortion on magnetotelluric data over a 3D regional structure, Geophys. J. Int., 132, 295-301, 1998.

Ogawa, Y., Effective modeling of local and regional EM data. 15th Workshop on Electromagnetic Induction on the Earth. Cabo Frio (Brasil) 2000. Invited Review Papers, pp. 259-265, 2000.

Utada, H. and H. Munekane, On galvanic distortion of regional threedimensional magnetotellurics impedances, Geophys. J. Int., 140, 385398, 2000.

Wannamaker, P. E., J. A. Stodt, and L. Rijo, A stable finite element solution for two-dimensional magnetotelluric modeling, Geophys. J. R. Astr. Soc., $\mathbf{8 8}, 277-296,1986$.

J. Ledo (e-mail: ledo@cg.nrcan.gc.ca), A. Gabàs (e-mail: agabas@geo. ub.es), and A. Marcuello (e-mail: alex@geo.ub.es) 